PTU-181 OPTIMISED RESPONSE PREDICTION IN OESOPHAGOGASTRIC ADENOCARCINOMAS (OGA) WITH COMBINATION OF MOLECULAR BIOMARKERS, SERUM CELL DEATH MARKERS AND FDG-PET

doi:10.1136/gutjnl-2012-302514c.181

${ }^{1} \mathrm{G} H$ Bain, ${ }^{*}{ }^{2} \mathrm{E}$ Collie-Duguid, ${ }^{2} \mathrm{G}$ Murray, ${ }^{2} \mathrm{~F}$ Gilbert, ${ }^{2} \mathrm{~A}$ Denison, ${ }^{1} \mathrm{~F}$ McKiddie, ${ }^{1} \mathrm{~T}$ Ahearn, ${ }^{1} \mathrm{~J}$ Leeds, ${ }^{1} \mathrm{P}$ Phull, ${ }^{1} \mathrm{~K}$ Park, ${ }^{2} \mathrm{~A}$ Welch, ${ }^{1} \mathrm{~L}$ Schweiger, ${ }^{2} \mathrm{R}$ D Petty. ${ }^{1}$ Aberdeen Royal Infirmary, Aberdeen, UK; ${ }^{2}$ University of Aberdeen, Aberdeen, UK

Introduction Predictive biomarkers (BMs) for OGA would optimise treatment selection and avoid ineffective therapy. Metabolic response (MR) defined as $>35 \%$ decrease in tumour FDG Standardised Uptake Value (SUV) between day 0 and day 14 after starting chemotherapy has a high negative predictive value (95\%) for response, but limited positive predictive value $(50 \%)$. Combining molecular BMs and serum cell death markers with FDG-PET may optimise response prediction. We used global gene expression profiling (GEP) and cell death ELISAs to identify molecular BMs and serum markers that when combined with FDG-PET would improve predictive accuracy.

Methods 28 patients with locally advanced/metastatic OGA received platinum based chemotherapy (PBC). FDG-PET scans were at day 0 \& 14 and GEP (Affymetrix ST1.0 Exon Genechips) on day 0 tumour biopsies. A tissue microarray comprising an independent set of 154 OGA who had surgery +/-neoadjuvant PBC was used with immunohistochemistry (IHC) for qualification of GEP results. Cytokeratin 18 (CK18) M30 (apoptosis) and M65 (apoptosis + necrosis) ELISAs (Pevivia, Sweden) were used to assess cell death from serial serum samples during chemo. Radiological response was assessed after $3 / 4$ cycles of PBC by RECISTv1.1.

Results We identified a gene expression signature (86 genes) that separated FDG-PET MR patients ( $>35 \%$ fall SUV day $0-14$ ) into those that do and do not have a RECIST response. In cross validation this signature correctly predicted response in 14/14 metabolic responders (MRs). Pathway analysis on GEP data identified potential novel mechanisms of response including the Leptin pathway. Leptin mRNA was higher in FDG MRs who did not have a RECIST response compared to those that did $(p=0.026)$. In the independent set high Leptin protein by IHC was associated with lack of histopathologic response to neoadjuvant $\operatorname{PBC}(n=64, p=0.007)$. High Leptin expression also had a therapy independent prognostic effect with longer survival in the absence of histopathologic response or with no neoadjuvant $\mathrm{PBC}$ and in low Leptin patients poor survival was mitigated to a degree by neoadjuvant PBC. Serum CK18M30 decreased from day $0-14$ in MRs but in metabolic nonresponders (MNRs) there was a smaller fall or a rise $(p=0.021)$. Levels in MNRs did not change with subsequent chemo. In MRs levels continued to fall in RECIST responders but increased again in non-responders.

Conclusion Molecular biomarkers (Leptin in particular) and serum cell death markers combine with FDG-PET to optimise response prediction in OGA. Further investigation of this combined molecular, serum and imaging approach is warranted.

Competing interests None declared.

\section{PTU-182 PERCUTANEOUS RADIOLOGICAL GASTROSTOMY IN OESOPHAGEAL CANCER PATIENTS: A FEASIBLE AND SAFE ACCESS FOR NUTRITIONAL SUPPORT DURING MULTIMODAL THERAPY}

doi:10.1136/gutjnl-2012-302514c.182

${ }^{1} \mathrm{G}$ Piessen, ${ }^{* 1} \mathrm{~W}$ Tessier, ${ }^{1} \mathrm{~W}$ B Robb, ${ }^{1} \mathrm{~N}$ Briez, ${ }^{1} \mathrm{~A}$ Boschetto, ${ }^{2} \mathrm{O}$ Ernst, ${ }^{1} \mathrm{C}$ Mariette. ${ }^{1}$ Department of Digestive and Oncological Surgery, University Hospital Claude Huriez,
Lille, France; ${ }^{2}$ Department of Digestive Radiology, University Hospital Claude Huriez, Lille, France

Introduction Percutaneous endoscopic gastrostomy is not widely used in malnourished oesophageal cancer (OC) patients because of concerns about feasibility in frequently obstructive tumours, suitability of the stomach as an oesophageal substitute, and potential for metastatic inoculation. A percutaneaous radiological gastrostomy (PRG) could be an optimal alternative.

Methods Experience with PRG among 1205 consecutive patients presenting with OC from 2000 to 2010 in our department was retrospectively reviewed. PRG was proposed for malnourished patients for whom neoadjuvant chemoradiation was scheduled. PRG placement success rate and major (Dindo-Clavien $>$ II) related complications were analysed. A matched cohort analysis was then constructed in patients who underwent oesophagectomy with gastroplasty $(n=759)$ to evaluate the impact of PRG placement on suitability of the gastric pull-up and on postoperative course. From 76 resected patients with PRG (PRG group), 152 randomly selected controls without PRG (no PRG group) were matched 2:1 by gender, age, ASA grade, cTNM stage and neoadjuvant treatment delivery.

Results PRG placement was planned in $269(22.3 \%)$ patients mainly with locally advanced OC (63.8\%). PRG placement was feasible in 259 (96.3\%) patients. 60-day PRG-related mortality and major morbidity rates were $0 \%$ and $3.8 \%$ respectively. For resected patients with gastroplasty, the PRG and no PRG groups were comparable regarding perioperative characteristics except for malnutrition more frequent in the PRG group $(p<0.001)$. At the time of operation, PRG takedown and site closure were uncomplicated and the use of the stomach was possible in all 76 patients. Despite higher malnutrition rate at presentation in the PRG group, overall and oesophageal surgery related morbidity rates were similar between the two groups ( $\mathrm{p}>0.432)$.

Conclusion PRG is feasible, safe and useful in non-selected patients with OC and does not compromise the suitability of the stomach as an oesophageal substitute in patients deemed to be resectable.

Competing interests None declared.

\section{PTU-183 INEFFECTIVENESS OF 18F-FLUORODEOXYGLUCOSE POSITRON EMISSION TOMOGRAPHY IN THE EVALUATION OF TUMOUR RESPONSE AFTER COMPLETION OF NEOADJUVANT CHEMORADIATION IN OESOPHAGEAL CANCER}

doi:10.1136/gutjnl-2012-302514c.183

${ }^{1,2,3} \mathrm{G}$ Piessen, ${ }^{*} \mathrm{~W}$ B Robb, ${ }^{2,4} \mathrm{G}$ Petyt, ${ }^{2,5} \mathrm{~A}$ Duhamel, ${ }^{2,6} \mathrm{X}$ Mirabel, ${ }^{2,3,4} \mathrm{D}$ Huglo, ${ }^{1,2,3} \mathrm{C}$ Mariette. ${ }^{1}$ Department of Digestive Surgery, Lille University Hospital, Lille, France, ${ }^{2}$ University of Lille - Nord de France, Lille, France; ${ }^{3}$ Inserm, UMR837, JeanPierre Aubert Research Center, Team 5, Lille, France; ${ }^{4}$ Department of Nuclear Medicine, Lille University Hospital, Lille, France; ${ }^{5}$ Department of Statistics, Lille University Hospital, Lille, France: ${ }^{6}$ Academic Radiotherapy Department, CLCC Oscar Lambret Comprehensive Cancer Center, Lille, France

Introduction After primary CRT, a non-invasive evaluation of the tumour response could help in the treatment decision to identify patients who may benefit from surgery. Whether FDG-PET provides clinically relevant information remains questionable. The objective of this prospective trial was to evaluate the role of $18 \mathrm{~F}$-Fluorodeoxyglucose-positron emission tomography (FDG-PET) in the assessment of tumour response after the completion of neoadjuvant chemoradiation (CRT) in patients with locally advanced resectable oesophageal cancer.

Methods Operable patients with locally advanced oesophageal cancer (clinically staged T3 N0-1 M0) were enrolled. The complete 